\title{
Interpreting Internal Activation Patterns in Deep Temporal Neural Networks by Finding Prototypes
}

\author{
Sohee Cho* \\ Graduate School of AI, KAIST \\ Seongnam, Republic of Korea \\ sohee.cho@kaist.ac.kr \\ Ginkyeng Lee \\ Ulsan National Institute of Science and Technology \\ Ulsan, Republic of Korea \\ gin908@unist.ac.kr
}

\author{
Wonjoon Chang* \\ Graduate School of AI, KAIST \\ Seongnam, Republic of Korea \\ one_jj@kaist.ac.kr \\ Jaesik Choi ${ }^{\dagger}$ \\ Graduate School of AI, KAIST \\ Seongnam, Republic of Korea \\ INEEJI \\ Seongnam, Republic of Korea \\ jaesik.choi@kaist.ac.kr
}

\begin{abstract}
Deep neural networks have demonstrated competitive performance in classification tasks for sequential data. However, it remains difficult to understand which temporal patterns the internal channels of deep neural networks capture for decision-making in sequential data. To address this issue, we propose a new framework with which to visualize temporal representations learned in deep neural networks without hand-crafted segmentation labels. Given input data, our framework extracts highly activated temporal regions that contribute to activating internal nodes and characterizes such regions by prototype selection method based on Maximum Mean Discrepancy. Representative temporal patterns referred to here as Prototypes of Temporally Activated Patterns (PTAP) provide core examples of subsequences in the sequential data for interpretability. We also analyze the role of each channel by Value-LRP plots using representative prototypes and the distribution of the input attribution. Input attribution plots give visual information to recognize the shapes focused on by the channel for decision-making.
\end{abstract}

\section{CCS CONCEPTS}

- Computing methodologies $\rightarrow$ Temporal reasoning.

\section{KEYWORDS}

Temporal Convolutional Neural Network, Interpretability, Temporally Activated Pattern, Prototype Selection, Input Attribution

\footnotetext{
"Both authors contributed equally to this research.

${ }^{\dagger}$ Corresponding Author
}

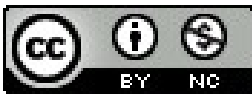

This work is licensed under a Creative Commons Attribution-NonCommercial International 4.0 License.

KDD '21, August 14-18, 2021, Virtual Event, Singapore.

(C) 2021 Copyright held by the owner/author(s).

ACM ISBN 978-1-4503-8332-5/21/08.

https://doi.org/10.1145/3447548.3467346

\section{ACM Reference Format:}

Sohee Cho, Wonjoon Chang, Ginkyeng Lee, and Jaesik Choi. 2021. Interpreting Internal Activation Patterns in Deep Temporal Neural Networks by Finding Prototypes. In Proceedings of The Association for Computing Machinery's Special Interest Group on Knowledge Discovery and Data Mining (KDD 2021). ACM, NY, NY, USA, 9 pages. https://doi.org/10.1145/3447548.3467346

\section{INTRODUCTION}

The amount of temporal data has increased greatly due to the use of efficient and diverse automatic information systems such as manufacturing sensors, stock database systems and wearable healthcare devices. To utilize such vast amounts of temporal data, the demand for applications of deep learning models in industry has grown rapidly. However, most industrial fields require transparency in decision-making when using deep learning models. Thus, they are still hesitant to adopt AI systems due to the lack of interpretability in their internal processes.

Currently, many AI researchers are attempting to understand the decision processes of deep learning models. Interpretable artificial intelligence methods explain and interpret decisions of complex systems with illustrative or textual descriptions [13, 23]. These approaches for deep learning are categorized into explaining input attribution methods based on the relevance score [6, 18-20], gradient-based methods [24-26], explaining internal nodes methods $[3,4]$, explaining through attention methods [9, 14], examplebased explanation methods $[5,15,16]$ and generating explanation methods [1].

However, these methods mainly focus on the image domain, and there have been few efforts to apply interpretation techniques to time series data. Objects in images can easily be recognized visually, because there is a considerable amount of human-annotated segmentation information for image datasets. However, there is not such information provided for most time series datasets. Thus, it is challenging to find semi-global shapes of the type a neural network can detect in time series input due to a lack of temporally segmented annotation data.

To address this issue, we suggest a new framework that visualizes representative temporal patterns by building a prototype selection method and identifying what the neural networks detect 


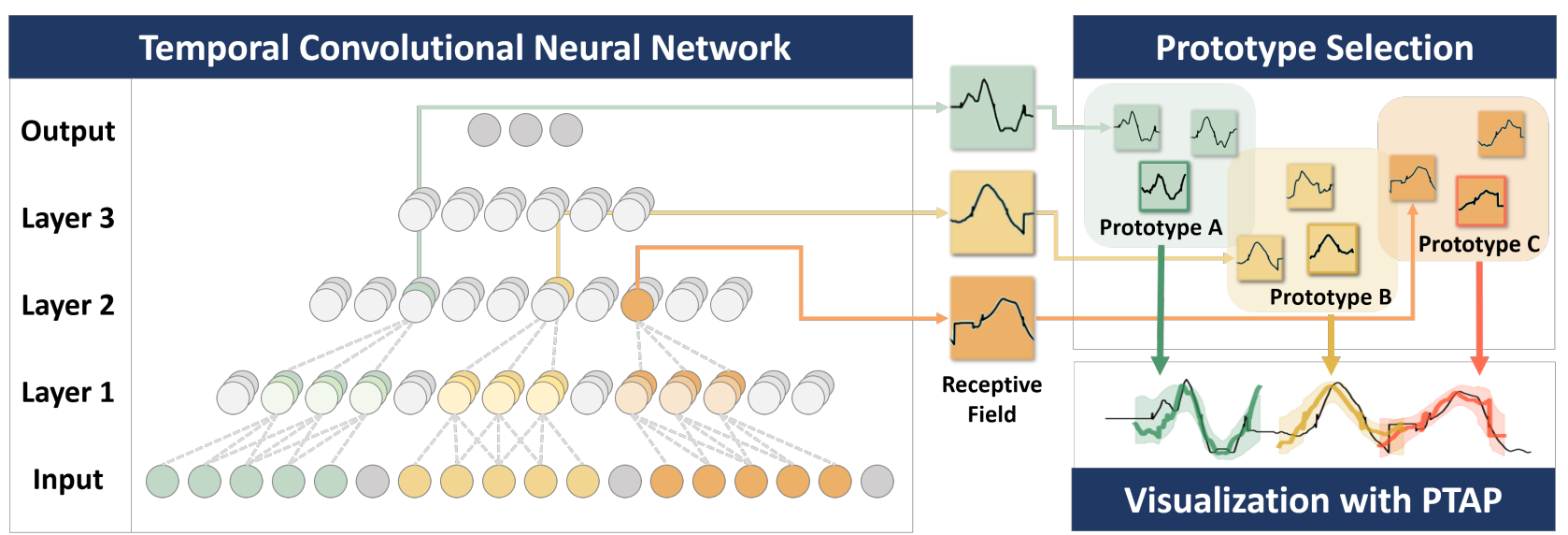

Figure 1: Our proposed framework, PTAP (Prototypes of Temporally Activated Patterns), extracts highly activated periods from activation maps of a temporal convolutional neural network, and categorizes highly activated periods using prototype selection method, and then visualizes those patterns on the original inputs. By doing so, it identifies temporal patterns activating each channel most and makes users to easily verify which patterns are commonly observed from trained data.

for decision-making in sequential data. Our framework makes the following contributions:

- Without handcrafted segmentation labels, our framework provides representative subsequences that activate channels of a convolutional neural network the most.

- We improve an existing greedy prototype selection method by constructing the Gram kernel matrix with feature values in neural networks instead of the simple radial basis function kernel with input values. The prototype results are more reasonable for time series as our method is less sensitive to the shift of subsequences.

- With our value-attribution score plots that show the distribution of input attributions for each prototype group, our framework helps to explain the shapes focused on by the channel for decision-making purposes.

Figure 1 describes the entire procedure of our framework. We first find important subsequences, which are due to high activations, from the input sequences. Then, prototypes are selected as representative temporal patterns from detected subsequences. Finally, our framework provides insight into the process of internal channels in a neural network through visualization. This visualization shows general shapes recognized by the network with core points for decision-making. To the best of our knowledge, this is the first attempt to extract and visualize patterns that highly activate internal nodes and to analyze the role of channels with the distribution of the input attributions. This will help users to understand how deep neural networks learn time series data intelligibly.

\section{RELATED WORK}

Convolutional Neural Networks (CNNs) [17] can learn features from the input by considering the spatial correlation using filters. A partial region of the feature map for each channel receives information from only a restricted subset of the output of previous layers. This input subset, called a receptive field, represents what the channel captures from the input.
The CNN architecture is widely known to be useful for image domains, but it can also be utilized for time series data in order to extract local information from nearby data points which are sequentially correlated. In fact, Recurrent Neural Networks (RNNs), which share weight parameters over temporal steps, have been successfully used in natural language processing, where the data of interest have discrete values. However, CNN-based methods, such as ST-GCN [28] and WaveNet [22], have demonstrated outstanding performance on classification and regression tasks involving time series data with continuous values. Therefore, we select CNN-based models to determine the roles of channels.

Network dissection [3] is a means of quantifying the interpretability of the individual channels of deep CNNs in image domains. This method evaluates the degree of matching of highly activated feature maps and corresponding concepts from segmentation labels. This approach is not applicable to datasets without segmentation labels, such as most time series data. Thus, we suggest the extraction of prototypes without given labels in order to interpret individual channels for time series data.

Input attribution methods $[6,18-20]$ compute a relevance score for each input component to quantify its attribution to the activation value. We analyze the relevance score for temporal patterns to identify the shapes focused on by the channel in neural networks.

Prototype methods represent the data distribution by a set of points in the feature space. K-medoids is a simple prototype method, as it proposes a set of prototypes indicating representative samples of clusters in the feature space. There have been several attempts to improve the interpretability of highly complex distributions using an example-based explanation $[5,15,16]$. The MMD-critic method [16] uses a greedy algorithm with the maximum-mean discrepancy (MMD), which is a distance metric that evaluates the expected maximum difference between two distributions. We describe the details in Section 4. 


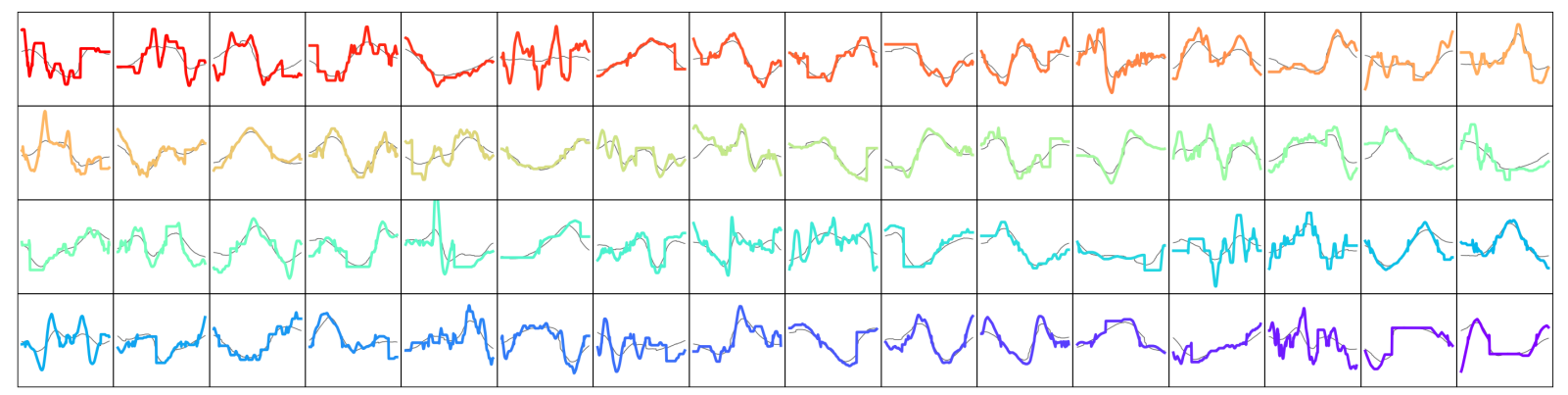

Figure 2: Each block shows the Prototype of Temporally Activated Patterns; colored lines refer to the prototype for the Uwave dataset and grey lines refer to the mean value of samples in the corresponding prototype group. Prototypes extracted from other datasets are presented in Figures 10 and 11.

\section{PROTOTYPES OF TEMPORALLY ACTIVATED PATTERNS (PTAP)}

This section describes the overall framework to visualize representative temporal patterns for given time series input. First we define and extract patterns detected by neural networks. Then, we select a set of prototypes that reflects the entire distribution of patterns. The details of the prototype selection method is provided in Section 4. Finally, we show how to understand the role of channels with prototypes and input attribution in Section 5.

\subsection{Temporally Activated Pattern (TAP)}

Analyzing highly activated parts of activation maps can play an important role in securing a better understanding of temporal patterns in neural networks. Our algorithm interprets the decisions of neural networks by extracting temporally activated nodes which have high activation value in a channel ${ }^{1}$ and visualizing the subsequences of the input data that contribute to the highly activated nodes.

Highly Activated means that certain nodes in a channel have larger activation values that exceed the channel's threshold. We compute a threshold $T_{j, k}$ that satisfies $P\left(a_{j, k}>T_{j, k}\right)=\theta$ to select highly activated units for each channel $k$ at layer $j$, where $T_{j, k}$ is the threshold of channel $k$ at hidden layer $j$, and $a_{j, k}$ is the distribution of the $k$ th channel activations at hidden layer $j$.

In channel $k$, we consider the highly activated node satisfying the condition $A_{j, k}[i]>T_{j, k}$ where $i$ is the temporal index of the node and $A_{j, k}[i]$ is the activation value of a node $i$. Let $H$ be a set of those indices that have high activations. We define a Temporally Activated Pattern (TAP) as the input receptive field of a node in $H$. This period is the important subsequence that we seek. We define Temporally Activated Unit (TAU) as the vector of activation values on the specific temporal index in $H$. That is, it denotes $\left[A_{j, 1}[i], \cdots, A_{j, c}[i]\right]^{T}$ where $i \in H$ and $c$ is the number of channels in layer $j$. Thus, the dimensionality of TAU is the same as the number of channels in layer $j$. The detailed process is described in Algorithm 1. $N_{j, k}$ in Algorithm 1 denotes the number of nodes in channel $k$ at hidden layer $j$.

${ }^{1}$ Network Dissection [3] refers to this channel as a unit. In this paper, it is necessary to distinguish between a channel and the basic elements in a channel. To do so, we refer to an activation map as a channel, one element on each channel as a node and a vector of nodes on the specific temporal position for the whole channels as a unit.

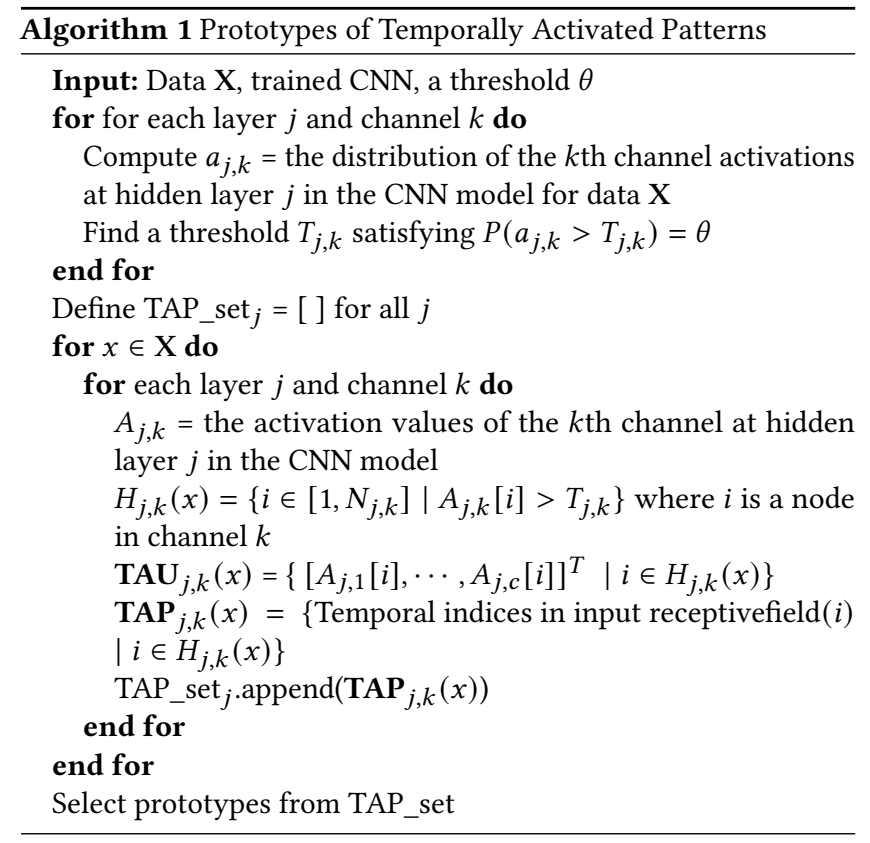

\subsection{Patternizing Representative Sub-sequences}

Thus far, we have found important subsequences by which to determine which individual channels in Deep Temporal CNNs (DTCNNs) are being examined in time series data. At this point, we characterize these subsequences and assign general shapes to them. In this paper, we build an improved prototype selection method (explained in Section 4) based on the Maximum Mean Discrepancy (MMD), which measures the discrepancy between two distributions, to characterize representative temporal patterns from all detected subsequences in a TAP.

Since deep learning models have multiple layers of nonlinearity, it is difficult to interpret the relationships between the inputs and the channel activation based only on the Euclidean distance [11, 15]. To solve this problem, example-based explanation methods attempt to select particular samples from the dataset to represent the underlying data distribution. The MMD-based prototype selection method is one of them. A particular sample, called a prototype, 
corresponds to a representative sample of the data. We improve the MMD-based prototype selection method by making it more effective and then define Prototypes of Temporally Activated Patterns (PTAP) as a set of prototypes from TAP_set. Note that the TAP_set should be defined according to the layer, as channels in different layers have different lengths of the input receptive field. In Figure 2, we visualize the selected prototypes from the third layer on the Uwave dataset.

\subsection{Visualization with PTAP}
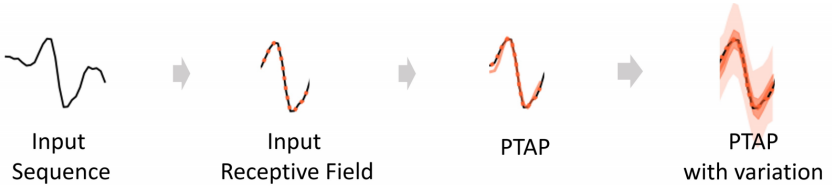

Figure 3: The process of visualization

We will describe how our framework provides interpretation with PTAP in Section 6.2. Figure 3 briefly explain the visualization process. The black line is the original input sequence, the colored dotted line is the input receptive field, which means the detected part. The colored line shows the PTAP and the colored shadow denotes the variation in the patterns from the same prototype group. Since the input receptive fields of the channel activations can have overlapped areas, we remove some overlapped patterns for PTAP to illustrate the important patterns, which have high similarity to the subsequences, more clearly.

\section{PROTOTYPES SELECTION}

This section briefly reviews the greedy prototype selection algorithm [16] based on Maximum Mean Discrepancy (MMD). Then, we introduce our improvements of the method to extract important subsequences from time series data.

\subsection{Maximum Mean Discrepancy}

MMD [12] is a distance metric that evaluates the expected maximum difference between two distributions $P$ and $Q$ over a function space $\mathcal{F}$ :

$$
\operatorname{MMD}(\mathcal{F}, P, Q)=\sup _{f \in \mathcal{F}}\left(\mathrm{E}_{x \sim P}[f(x)]-\mathrm{E}_{y \sim Q}[f(y)]\right)
$$

It has already been proved that given a reproducing kernel Hilbert space $\mathcal{F}$ with kernel function $k: \mathcal{X} \times \mathcal{X} \mapsto \mathbb{R}$ the supremum is achieved at:

$$
f(x)=\mathrm{E}_{x^{\prime} \sim P}\left[k\left(x, x^{\prime}\right)\right]-\mathrm{E}_{y^{\prime} \sim Q}\left[k\left(x, y^{\prime}\right)\right]
$$

Then, we easily obtain an unbiased finite sample estimate of the squared MMD in terms of kernel functions:

$$
\begin{aligned}
& \operatorname{MMD}^{2}(\mathcal{F}, X, Y) \approx \frac{1}{n^{2}} \sum_{i, j \in[n]} k\left(x_{i}, x_{j}\right) \\
& -\frac{2}{n m} \sum_{i \in[n], j \in[m]} k\left(x_{i}, y_{j}\right)+\frac{1}{m^{2}} \sum_{i, j \in[m]} k\left(y_{i}, y_{j}\right)
\end{aligned}
$$

where $X$ is a set of given $n$ samples from $P$ and $Y$ is a set of given $m$ samples from $Q$. A set of prototypes is selected to be similar to the original data distribution so that the MMD is close to zero.

\subsection{Conditions for Greedy Prototype Selection}

To find a set of prototypes, which is a subset of the entire dataset, to minimize the $\mathrm{MMD}^{2}$, it is necessary to maximize the following objective function:

$$
\begin{aligned}
& J_{b}(S)=\frac{1}{n^{2}} \sum_{i . j=1}^{n} k\left(x_{i}, x_{j}\right)-\operatorname{MMD}^{2}\left(\mathcal{F}, X, X_{S}\right) \\
& =\frac{2}{n|S|} \sum_{i \in[n], j \in S} k\left(x_{i}, x_{j}\right)-\frac{1}{|S|^{2}} \sum_{i, j \in S} k\left(x_{i}, x_{j}\right)
\end{aligned}
$$

where $S \subseteq[n]$ is a subset of all the indices and $X_{S}=\left\{x_{i} \mid i \in S\right\}$.

This set optimization may be quite complex since there are numerous combinations to construct subsets. We can relax the optimization problem by a greedy selection algorithm. When the objective is a monotone submodular function, a greedy algorithm achieves at least a constant fraction of the optimal solution [21]. Therefore, we can efficiently select a set of prototypes if $J_{b}(S)$ is a monontone submodular set function.

The greedy algorithm selects the sample index $i \in[n] \backslash S$ that maximizes $J_{b}(S \cup i)-J_{b}(S)$ for each iteration. After selecting a set of prototypes, each sample is clustered to one prototype that has the largest kernel value, which means they are very similar.

The monotone submodularity of the set function (4) depends on the kernel matrix [16]. Let the kernel matrix $\mathrm{K} \in \mathbb{R}^{n \times n}$ be element-wise non-negative, with equal diagonal terms $k_{i, i}=k_{*}>0$ for all $i \in[n]$, and be diagonally dominant. The monotone submodularity requires all off-diagonal terms $k_{i, j}$ to be smaller than $k_{*} /\left(n^{3}+2 n^{2}-2 n-3\right)$. That is, the kernel matrix should be nonnegative, have constant diagonal terms and have very small offdiagonal terms.

\subsection{Improved Prototype Selection}

Originally, the greedy prototype selection method based on MMD finds prototypes with the radial basis function kernel (rbf kernel) in the input space. The rbf kernel $k_{i, j}=\exp \left(-\gamma\left\|x_{i}-x_{j}\right\|_{2}^{2}\right)$ with a large value of $\gamma$ satisfies the conditions for monotone submodularity described in Section 4.2. However, a large value of $\gamma$ makes the algorithm vulnerable to the temporal shift of samples, which hinders distinguishing true shapes of subsequences. Furthermore, input values may not sufficiently reflect the characteristics of internal patterns enough.

Thus, we build prototypes based on the features in the internal layer, which we defined as Temporally Activated Units (TAUs) in Section 3. We construct the Gram kernel matrix of TAUs for prototype selection rather than using the rbf kernel. TAUs are robust to the temporal shift due to the structure of convolution and pooling, so we expect that TAUs represent necessary features for decision-making of the DTCNN.

Let $\phi: \mathbb{R}^{d} \mapsto \mathbb{R}^{c}$ be a function that projects the TAP to the standardized TAU where $d$ is a dimensionality of pattern subsequences and $c$ is the number of channels in the target layer. For $x \in$ TAP_set, the Euclidean norm of $\phi(x)$ should be one so that $k(x, x)=\phi(x)^{\mathrm{T}} \phi(x)=1$. Then, we obtain the Gram matrix $\mathbb{K}$ with $k_{i, j}=k\left(x_{i}, x_{j}\right)=\phi\left(x_{i}\right)^{\mathrm{T}} \phi\left(x_{j}\right)$. It is a valid kernel matrix with equal diagonal terms $k_{i, i}=1$ and off-diagonal terms $0 \leq k_{i, j}<1$. Note 
that the use of ReLU activation function guarantees element-wise non-negativity of this Gram kernel matrix.

We suggest to use the Gram kernel matrix, which is positive standardized, as the kernel for (4). Actually, this matrix may not satisfy the diagonal dominance, which means that the greedy solution may not be close to the optimal solution. We encourage the diagonal dominance of the Gram kernel matrix by using powers of the kernel function. Note that the kernel function $k^{n}\left(x_{i}, x_{j}\right)$ is a valid kernel as it is shown that the multiplication of valid kernels is also valid. Then, we can reduce the off-diagonal terms to be close to zero by using powers of the Gram matrix kernel. It keeps the order of the similarity between patterns as the original Gram matrix.

\section{ANALYZING THE SENSITIVITY OF CHANNEL ACTIVATION}
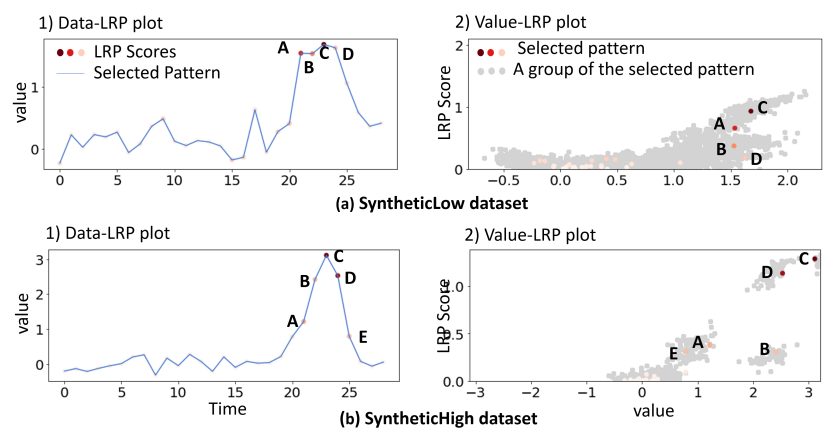

Figure 4: The column on the left shows the subsequences (patterns) with blue lines, and the column on the right shows the sensitivity of the relevance scores to changes in the input values. The gray points are individual data points. In both columns, red points are the LRP scores. The more red that is visible, the higher the relevance score is.

To understand the relationships among the activations of channels and the extracted patterns, we compute the distribution of the input attributions with respect to each channel. In other words, our goal is to determine which changes in the pattern shape affect more or less the channel's activation. We use Layer-wise Relevance Propagation (LRP) [19] to compute and calculate how much a change in a temporal position affects the channel activation.

The relevance scores of inputs become gradients multiplied by the inputs when the ReLU is used as a non-linear function [19]. This idea is recursively applied to the previous layers from the output to decompose the relevance score into the sum of the relevance scores of the nodes in the internal layers [2]. Through this decomposition, we assign the relevance score to each data point from the channel activation so that the relevance scores act as the sensitivity measure.

We propose the Data-LRP plots and the Value-LRP plots to identify sensitive parts in terms of the channel activations. The DataLRP shows the subsequence (pattern) with the relevance score of each time point. The Value-LRP plot visualizes the LRP distribution where the $\mathrm{x}$-axis is the input value of the data point and the $\mathrm{y}$-axis is the relevance score. Therefore, the Value-LRP plot shows the sensitivity of channel activation to changes in input values.
We generate simple time series datasets, SyntheticLow and SyntheticHigh which have a single peak with low values and high values respectively. As shown in Figure 4 (b), the point $\mathbf{B}$ has a higher input value than $\mathbf{A}$, but the LRP score of $\mathbf{B}$ is smaller than that of $\mathbf{A}$. Even though $\mathbf{B}$ is located around $\mathbf{C}$ and $\mathbf{D}$, the LRP scores of $\mathbf{B}$ is much smaller than $\mathbf{C}$ and $\mathbf{D}$. That is, we can conclude that the channel focuses on more on decreases after peaks such as $\mathbf{C}$ and $\mathbf{D}$ compared to increases before peaks such as $\mathbf{A}$ and $\mathbf{B}$. In the case of (a), however, the channel focuses on increasing points compared to the previous time step, such as $\mathbf{A}$ and $\mathbf{C}$.

To the best of our knowledge, this is the first method to present the distributions of attributions for individual channels in a temporal neural network. These distributions show the different relevance changes based on the input shape and location. We apply this analysis method to a real world time series dataset in Section 6.4.

\section{EXPERIMENTAL RESULTS}

This section presents the experimental results for our framework. Details of the experimental settings including the time series datasets are explained. Then, the quantitative results and various qualitative analysis are presented.

Table 1: Description of Time Series Datasets

\begin{tabular}{cccccc}
\hline & $\begin{array}{c}\text { Time } \\
\text { Length }\end{array}$ & $\begin{array}{c}\text { Input } \\
\text { Sensor }\end{array}$ & Class & $\begin{array}{c}\text { Train } \\
\text { Size }\end{array}$ & $\begin{array}{c}\text { Test } \\
\text { Size }\end{array}$ \\
\hline Uwave $^{2}$ & 945 & 1 & 9 & 3582 & 896 \\
FordA & 500 & 1 & 2 & 3601 & 1320 \\
Starlight $^{3}$ & 1024 & 1 & 3 & 1000 & 8236 \\
HAR & 128 & 9 & 6 & 7352 & 2947 \\
\hline
\end{tabular}

Table 2: Filters in DTCNN models

\begin{tabular}{lccc}
\hline DATASET & L1 & L2 & L3 \\
\hline UWAVE, STARLIGHT & 15 & 11 & 7 \\
FordA, HAR & 7 & 5 & 3 \\
\hline
\end{tabular}

Table 3: Filters in ResNet models

\begin{tabular}{llllllllll}
\hline & L1 & L2 & L3 & L4 & L5 & L6 & L7 & L8 & L9 \\
\hline UWAVE & 15 & 11 & 7 & 15 & 11 & 7 & 15 & 11 & 7 \\
HAR & 8 & 5 & 3 & 8 & 5 & 3 & 8 & 5 & 3 \\
\hline
\end{tabular}

\subsection{Experiment Settings}

We use time series datasets of UCR repository [8]; Uwave (UWaveGestureLibraryAll), FordA and Starlight (StarlightCurves). And Smartphone Dataset for Human Activity Recognition (HAR) [10] is a smartphone sensor dataset that records a person performing eight different activities. In this paper, we mainly use Uwave dataset, but other 
datasets show similar results. Table 1 includes detail information of datasets used in our experiments.

We use a temporal CNN which is composed of three convolution layers followed by pooling layers, and one fully connected layer. The ReLU function is used as the activation function in each hidden layer. The batch size is 64 and 500 training epochs are used. We also apply our framework to ResNet [27] with nine deeper layers. Figures 5 and 6 show the PTAP results for various models and datasets. The length of filters in each layer is presented in Tables 2 and 3.

In Section 3.2, there is a trade-off in the threshold $T_{j, k}$ between the accuracy and the degree to which it can be explained when selecting highly activated nodes. More specifically, we consider the set of activated nodes that maintains the accuracy of the original model. This technique is widely used in explainable AI as the smallest support set [7]. As an example in the third layer, when we have the top 5\% of highly activated nodes, the prediction accuracy is maintained at $73 \%$, while having the top $2.5 \%$, top $10 \%$ and top $20 \%$ nodes will result in corresponding accuracy rates of $60 \%, 81 \%$, and $88 \%$. In our experiments, a set of the top 5\% highly activated nodes is a reasonably small set of representative nodes of the model while maintaining in general the original prediction accuracy rate. Our choice of threshold is similar with previous work in deep neural networks for visual recognition [3]. In our experiments, the number of prototype $m$ set as 64 for the Uwave dataset, HAR, and FordA. For the Starlight dataset, we make 32 prototypes empirically.

\subsection{Analysis of Internal Features of Various DTCNN Models}

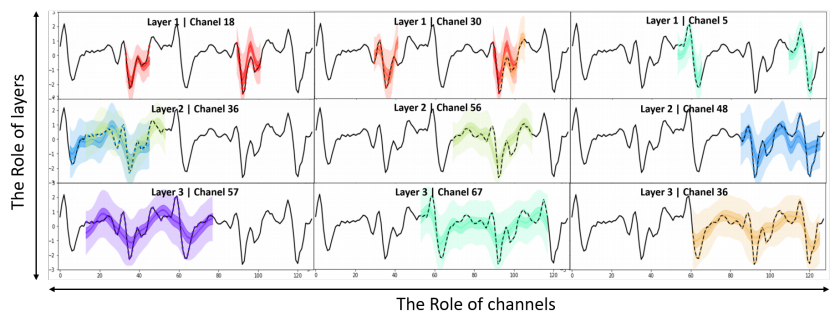

Figure 5: [PTAP Result in CNN Model, HAR Data 1247 Sensor 1]

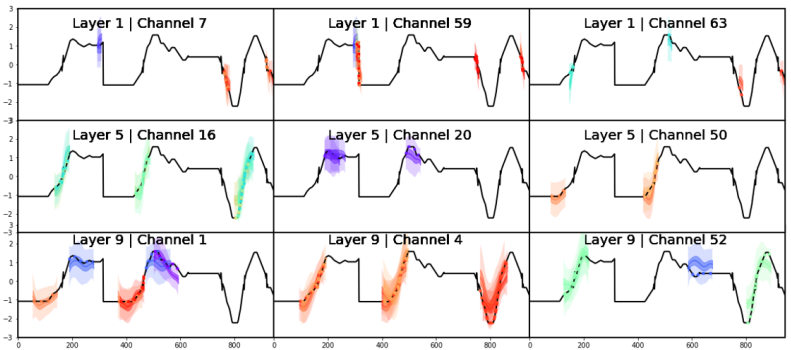

Figure 6: [PTAP Result in ResNet Model, UWave Data 571, Sensor 0]
Our framework visualizes temporal representations of extracted from different channels and layers of various temporal deep neural networks in Figures 5 and 6. We visualizes the variation of patterns, as described in Section 3.3. The x-axis shows the patterns from the different layers and the y-axis shows the role of the hidden layer.

Figure 5 shows that the high-level layers, such as Channel 57 in Layer 3, ignore local fluctuations and capture the long-term trend. However, the low-level layers are affected by detailed features. We visualize representative patterns in the case of ResNet in Figure!6. The channels in the lower layers like Channels 7 and 59 of Layer 1 tend to focus on rapid changes or inflection points, while the channels in the higher layers recognize extreme changes in softer patterns.

\subsection{Quantitative Results of Prototype Selection}

To verify that the selected prototypes reflect the representative subsequences of the dataset, we train a logistic regression model to test if the weak classifier can classify the label with only the existence of each type of the prototype for the given sequence. Table 4 shows logistic regression accuracies with the different prototype selection methods. Thus, the input of each sequence is a multi-hot vector $v$ so that $v_{i}=1$ if the $i$ th prototype is observed in the given sequence and $v_{i}=0$ if not. We use K-medoids and MMD prototype selection with different kernels. RBF denotes the use of the rbf kernel with $\gamma=0.03$, GRAM denotes the use of the Gram kernel matrix and $\mathrm{GRAM}^{P}$ denotes the use of powers of the Gram kernel matrix, in this case we use $P=20 . M$ is the number of prototypes. We trained 30 classifiers with distinct train and test sets and describe the mean and standard deviation of accuracies on Table 4.

Table 4: Logistic Regression accuracies using each set of prototypes from different selection algorithms

\begin{tabular}{ccccc}
\hline $\mathrm{m}$ & K-medoids & RBF & Gram & Gram $^{P}$ \\
\hline 8 & $33.6 \pm 0.8$ & $32.4 \pm 1.3$ & $41.4 \pm 1.1$ & $39.5 \pm 1.0$ \\
10 & $42.7 \pm 1.4$ & $37.8 \pm 1.6$ & $44.7 \pm 1.4$ & $42.8 \pm 1.5$ \\
20 & $57.9 \pm 1.2$ & $53.0 \pm 1.4$ & $54.7 \pm 1.5$ & $57.5 \pm 1.5$ \\
30 & $61.5 \pm 1.1$ & $59.8 \pm 1.2$ & $61.5 \pm 1.4$ & $63.3 \pm 1.3$ \\
40 & $65.3 \pm 1.1$ & $65.2 \pm 1.6$ & $63.9 \pm 1.3$ & $66.2 \pm 1.4$ \\
50 & $66.1 \pm 1.6$ & $69.5 \pm 1.4$ & $68.3 \pm 1.5$ & $69.7 \pm 1.5$ \\
60 & $68.5 \pm 1.1$ & $71.2 \pm 1.1$ & $69.9 \pm 1.1$ & $69.7 \pm 1.3$ \\
70 & $67.7 \pm 1.1$ & $71.9 \pm 1.1$ & $70.6 \pm 1.1$ & $72.4 \pm 1.2$ \\
\hline
\end{tabular}

GRAM and $\mathrm{GRAM}^{P}$ outperform K-medoids and RBF in many cases. RBF has lower accuracies with the small number of the prototypes even though the matrix satisfies diagonal dominance more strongly than GRAM, which suggests that our Gram kernel matrix based selection is better separating subsequences that are necessary for decision-making. However, GRAM has relatively low accuracies with the large number of prototypes since the diagonal dominance of the kernel matrix of a prototype set can be violated. That is, the greedy selected set of prototypes may not be close to the optimal set of prototypes. We can use $\mathrm{GRAM}^{P}$ to obtain improved classification accuracies. In fact, GRAM $^{P}$ has the best accuracy when using 70 prototypes. It is similar to adjusting $\gamma$ of the rbf kernel. 


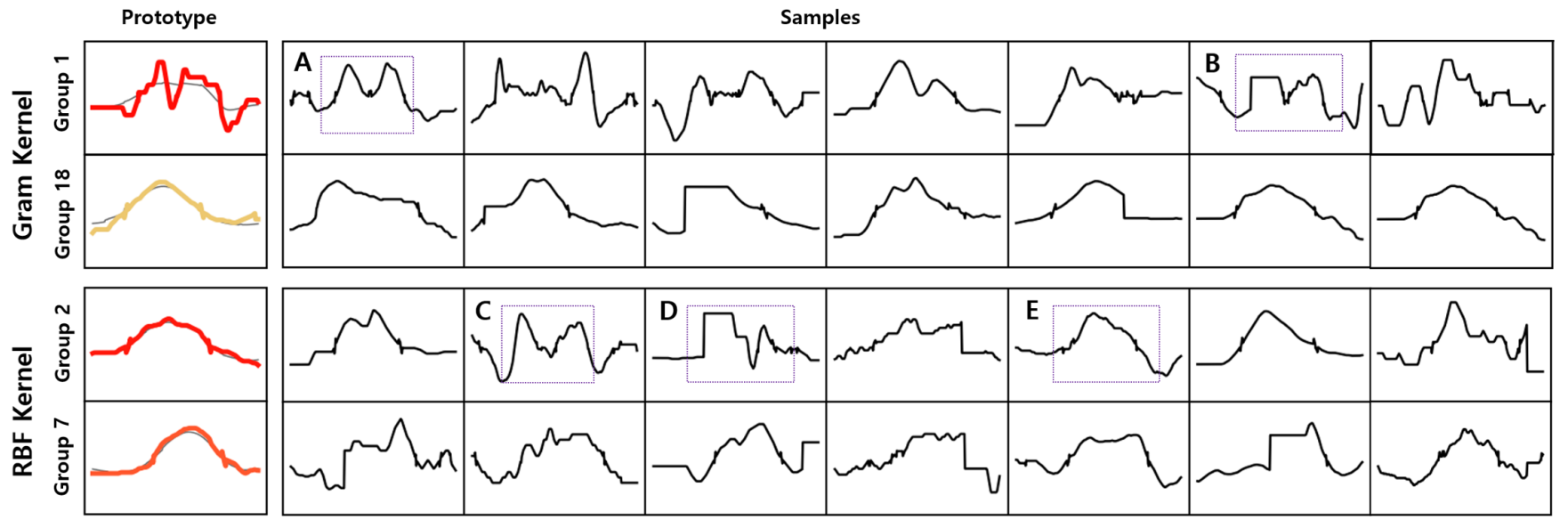

Figure 7: Prototypes extracted by the MMD method based on the Gram kernel and the rbf kernel in Uwave dataset. The first column shows the prototypes (representative samples) of selected groups and the remaining columns show which subsequences belong to the given prototype groups. We observe that the prototypes selected from the Gram kernel reflect the shapes of subsequences better since the Gram kernel is robust on the temporal shift of the pattern. Note that Group 2 and its temporally shifted one (Group 7) are extracted by the rbf kernel based method as distinct ones.

\subsection{Qualitative Analysis with Extracted Prototypes}

We observe how different the selected prototypes are in the Uwave dataset. In both cases, we build 64 prototypes from trained DTCNNs and choose subsequences that belong to each prototype. The similarity is computed by the kernel function values. Figure 7 shows the difference in prototype results with samples between the rbf kernel matrix and the Gram kernel matrix. We select two prototypes for each case so that four prototype groups have the similar mean shapes, which are bell-like shapes. For each prototype group, the colored line denotes the prototype and the grey line denotes the mean shape of samples belonging to that prototype group in the first column. Even though those groups have similar bell-like mean shapes, the actual subsequences in each group have quite different properties.

We can easily identify that Group 1 has samples with M-like patterns and Group 18 has samples with a single peak. Our method captures the M-like patterns as a distinct group, Group 1. It seems reasonable that $\mathbf{A}$ and $\mathbf{B}$ belong to the single group as shown in Figure7. On the other hand, when we obtain prototypes of Group 2 and Group 7 selected from the rbf kernel as in the existing MMD based prototype selection [16], a decision about which group a given sample belongs to seems to be ambiguous. $\mathbf{C}$ and $\mathbf{D}$ are respectively similar to the case of $\mathbf{A}$ and $\mathbf{B}$. However, the prototype of Group 2 has a bell-like shape, not an M-like shape. The rbf kernel evaluates the similarity between two samples based on the Euclidean distance. That is, the subsequence may be classified to the group whose prototype has a different shape if its average deviation is small.

Furthermore, the rbf kernel is sensitive to the temporal shift. E seems close to the prototype of Group 7. However, this sample is classified as Group 2 since the similarity computed by the rbf kernel grows rapidly as the shape shifts sideways. It is not desirable to choose the multiple prototypes that have similar shapes like the prototypes of Group 2 and Group 7 in the rbf kernel case. Our method uses the Gram matrix based on feature values so that we can select prototypes robust to the temporal shift.

\subsection{Sensitivity Analysis with Value-LRP Plots}

To demonstrate the benefits of our Value-LRP plot, we perform a sensitivity analysis with the Uwave dataset, as an example. The left column in Figure 8 (b) shows the Data-LRP and Value-LRP plot of Pattern 6685. Since Pattern 6685 belongs to Group 22 in Channel 4 , the gray points in the Value-LRP plot refer to another pattern, i.e., pattern 4740 . Pattern 6685 resembles its prototype in Figure 8 (a); the starting part has a fluctuating shape, the middle part has a stepped shape, and the end part has a small convex curve. The distribution in the Value-LRP plot usually shows upward curves. In general, values which are far from the mean have higher LRP scores, one example being A. However, there are many data and pattern specific cases well captured by our Value-LRP Plots. Pattern 4740 is similar to its prototype, Group 22, but its scales are quite different. Especially in the middle part, the length is shorter than that of the prototype, but the height is taller compared to the prototype. Moreover, $\mathbf{C}$ shows a drastic drop, causing the distribution to show a slight decrease in the Value-LRP plot.

Pattern 50284 has a shape similar to that of its prototype, Group 25 , and an upward curve in the value-LRP plot, while pattern 44617 shows some vertical spines, such as $\mathbf{I}, \mathbf{H}$, and $\mathbf{G}$. We surmise that these spines appear because the Data-LRP plot shows different LRP values for similar pattern values. For instance, $\mathbf{I}$ in the Data-pattern plot shows pattern values near -1.5 , but the corresponding LRP values vary from light pink to dark red. On the other hand, $\mathbf{H}$ and G show relatively less various LRP values from red to light pink. That is, when certain points in the pattern go to the top, the LRP scores increase. However, after the points reach the bottom, the LRP scores decrease gradually. 

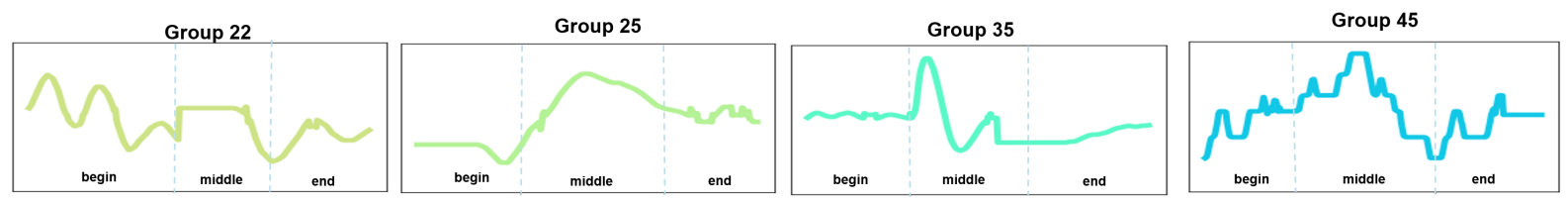

(a) Prototypes
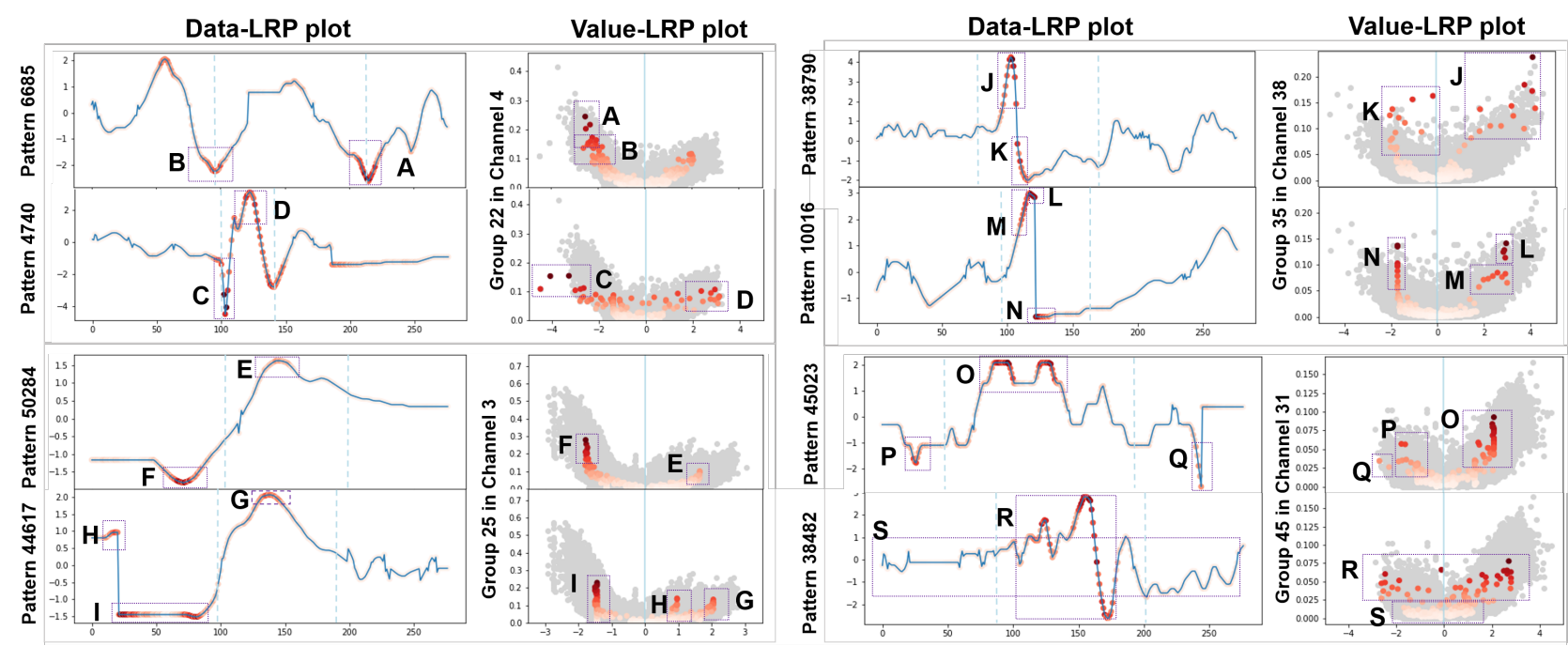

(b) Data-LRP plot and Value-LRP plot

Figure 8: (a) Prototypes of some selected groups (b) Data-LRP plot; blue lines are selected patterns and red points are LRP scores, and the Value-LRP plot; the $x$-axis is the value and the $y$-axis is the attribution value, and the LRP score. Detailed descriptions and explanations are provided in Section 6.4.

Patterns 38790 and 10016 have an abnormal spire shape similar to that of $\mathbf{J}$ and $\mathbf{M}$, and the corresponding LRP scores do not increase gradually. Specifically for the Data-LRP plot in pattern 10016, the spire shape has two parts, $\mathbf{M}$ and $\mathbf{L}$, and its value-LRP plot is also therefore separated as well. A stepped line such as that of $\mathbf{P}$ in Pattern 45023's Data-LRP plot would cause a stepped distribution. For pattern 38428, the distributions appear to separate into two parts, $\mathbf{R}$ and $\mathbf{S}$, because the Data-LRP plot also shows two separate parts, $\mathbf{R}$ and $\mathbf{S}$.

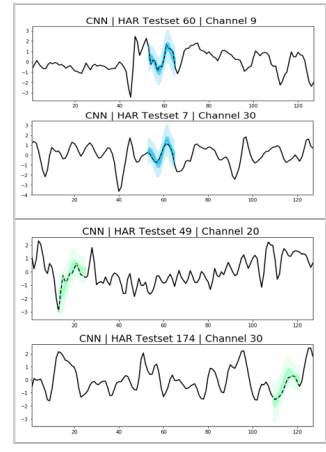

(a) Well-matched cases

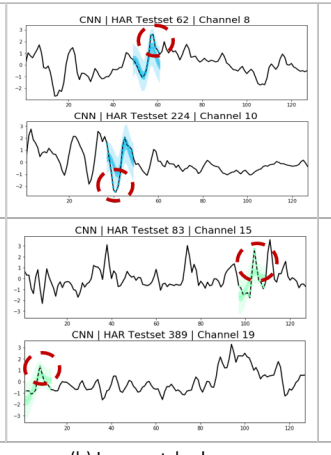

(b) Less-matched cases
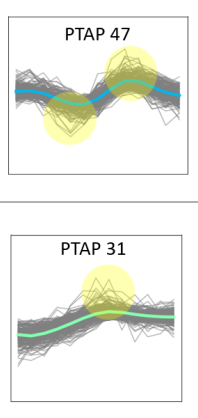

(c) PTAP

Figure 9: Comparisons between well-matched patterns and less well-matched patterns

\subsection{Analysis with Unseen Data}

Figure 9 illustrates how PTAP works well for the test dataset. The column on the left shows well-matched cases with new test data. On the other hand, the middle column shows examples of less wellmatched patterns through the red-dashed circles. Note that there are certain points where the actual data deviate from the assigned pattern for each less well-matched example. The positions of these points are reflected in the visualization of our framework. The column on the right shows that our visualization method successfully captures the positions with high uncertainty in the actual data.

\section{CONCLUSION}

We propose a new method to visualize representative temporal patterns in neural networks by breaking into the activations of the channels and analyzing their receptive fields in the input space. Our framework uses highly activated temporal regions and applies a clustering method to patternize these regions in order to obtain the general shapes without human-segmented information. We also propose the Gram kernel matrix using activations of channels for greedy prototype selection rather than using the radial basis function kernel. Consequently, our work can provide intuitive visualizations by the Value-LRP plot and PTAP and inspire people to understand AI systems in relation to real-world problems. 


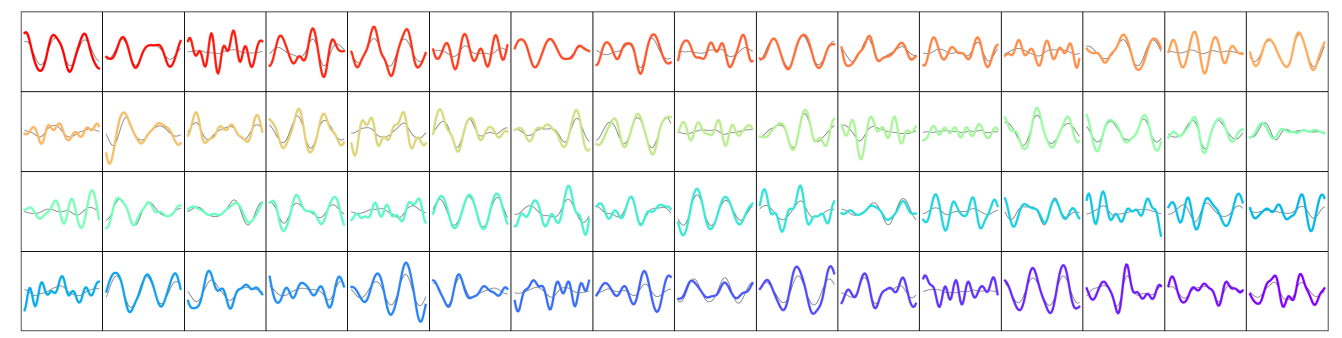

Figure 10: Prototypes extracted from FordA dataset

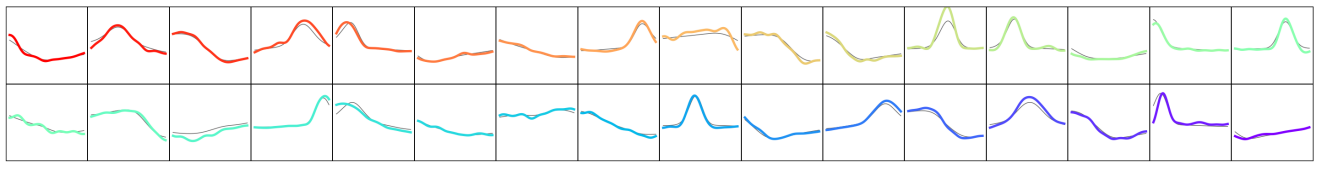

Figure 11: Prototypes extracted from Starlight dataset

\section{ACKNOWLEDGMENTS}

This work was supported by Institute for Information \& communications Technology Planning \& Evaluation (IITP) grant funded by the Korea government (MSIT) (No.2017-0-01779, XAI and No.20190-00075, Artificial Intelligence Graduate School Program (KAIST))

\section{REFERENCES}

[1] Jacob Andreas, Marcus Rohrbach, Trevor Darrell, and Dan Klein. 2016. Neural module networks. In Proceedings of the IEEE Conference on Computer Vision and Pattern Recognition. 39-48.

[2] Sebastian Bach, Alexander Binder, Gregoire Montavon, Frederick Klauschen, Klaus-Robert Muller, and Wojciech Samek. 2015. On Pixel-Wise Explanations for Non-Linear Classifier Decisions by Layer-Wise Relevance Propagation. PLOS ONE 10 (07 2015), 1-46. https://doi.org/10.1371/journal.pone.0130140

[3] David Bau, Bolei Zhou, Aditya Khosla, Aude Oliva, and Antonio Torralba. 2017 Network dissection: Quantifying interpretability of deep visual representations. In Proceedings of the IEEE conference on computer vision and pattern recognition. 6541-6549.

[4] David Bau, Jun-Yan Zhu, Hendrik Strobelt, Bolei Zhou, Joshua B Tenenbaum, William T Freeman, and Antonio Torralba. 2018. Gan dissection: Visualizing and understanding generative adversarial networks. arXiv preprint arXiv:1811.10597 (2018).

[5] Jacob Bien and Robert Tibshirani. 2011. Prototype selection for interpretable classification. The Annals of Applied Statistics 5, 4 (Dec 2011), 2403-2424. https: //doi.org/10.1214/11-aoas495

[6] Alexander Binder, Grégoire Montavon, Sebastian Lapuschkin, Klaus-Robert Müller, and Wojciech Samek. 2016. Layer-wise relevance propagation for neural networks with local renormalization layers. In International Conference on Artificial Neural Networks. Springer, 63-71.

[7] Chun-Hao Chang, Elliot Creager, Anna Goldenberg, and David Duvenaud. 2018. Explaining image classifiers by counterfactual generation. arXiv preprint arXiv:1807.08024 (2018)

[8] Yanping Chen, Eamonn Keogh, Bing Hu, Nurjahan Begum, Anthony Bagnall, Abdullah Mueen, and Gustavo Batista. 2015. The UCR Time Series Classification Archive. www.cs.ucr.edu/ eamonn/time series_data/.

[9] Edward Choi, Mohammad Taha Bahadori, Jimeng Sun, Joshua Kulas, Andy Schuetz, and Walter Stewart. 2016. Retain: An interpretable predictive model for healthcare using reverse time attention mechanism. In Advances in Neural Information Processing Systems. 3504-3512.

[10] Dheeru Dua and Casey Graff. 2017. UCI Machine Learning Repository. http: //archive.ics.uci.edu/ml

[11] Krishnamurthy Dvijotham, Robert Stanforth, Sven Gowal, Timothy A Mann, and Pushmeet Kohli. 2018. A Dual Approach to Scalable Verification of Deep Networks.. In UAI, Vol. 1. 2.

[12] Arthur Gretton, Karsten M Borgwardt, Malte J Rasch, Bernhard Schölkopf, and Alexander Smola. 2012. A kernel two-sample test. The fournal of Machine Learning Research 13, 1 (2012), 723-773.
[13] David Gunning, Mark Stefik, Jaesik Choi, Timothy Miller, Simone Stumpf, and Guang-Zhong Yang. 2019. XAI-Explainable artificial intelligence. Science Robotics 4, 37 (2019).

[14] Jay Heo, Hae Beom Lee, Saehoon Kim, Juho Lee, Kwang Joon Kim, Eunho Yang, and Sung Ju Hwang. 2018. Uncertainty-aware attention for reliable interpretation and prediction. In Advances in Neural Information Processing Systems. 909-918.

[15] Giyoung Jeon, Haedong Jeong, and Jaesik Choi. 2019. An Efficient Explorative Sampling Considering the Generative Boundaries of Deep Generative Neural Networks. arXiv preprint arXiv:1912.05827 (2019).

[16] Been Kim, Rajiv Khanna, and Oluwasanmi O Koyejo. 2016. Examples are not enough, learn to criticize! criticism for interpretability. In Advances in neural information processing systems. 2280-2288.

[17] Yann LeCun, Léon Bottou, Yoshua Bengio, and Patrick Haffner. 1998. Gradientbased learning applied to document recognition. Proc. IEEE 86, 11, 2278-2324.

[18] Hongzhi Li, Joseph G Ellis, Lei Zhang, and Shih-Fu Chang. 2018. Patternnet: Visual pattern mining with deep neural network. In Proceedings of the 2018 ACM on International Conference on Multimedia Retrieval. 291-299.

[19] Gregoire Montavon, Sebastian Lapuschkin, Alexander Binder, Wojciech Samek, and Klaus-Robert Muller. 2017. Explaining nonlinear classification decisions with deep Taylor decomposition. Pattern Recognition 65 (2017), 211 - 222. https: //doi.org/10.1016/j.patcog.2016.11.008

[20] Woo-Jeoung Nam, Jaesik Choi, and Seong-Whan Lee. 2019. Relative Attributing Propagation: Interpreting the Comparative Contributions of Individual Units in Deep Neural Networks. arXiv preprint arXiv:1904.00605 (2019).

[21] George L Nemhauser, Laurence A Wolsey, and Marshall L Fisher. 1978. An analysis of approximations for maximizing submodular set functions-I. Mathematical programming 14, 1 (1978), 265-294.

[22] Aaron van den Oord, Sander Dieleman, Heiga Zen, Karen Simonyan, Oriol Vinyals, Alex Graves, Nal Kalchbrenner, Andrew Senior, and Koray Kavukcuoglu. 2016. Wavenet: A generative model for raw audio. arXiv preprint arXiv:1609.03499 (2016)

[23] Wojciech Samek, Grégoire Montavon, Andrea Vedaldi, Lars Kai Hansen, and Klaus-Robert Müller. 2019. Explainable AI: interpreting, explaining and visualizing deep learning. Vol. 11700. Springer Nature.

[24] Ramprasaath R Selvaraju, Michael Cogswell, Abhishek Das, Ramakrishna Vedantam, Devi Parikh, and Dhruv Batra. 2017. Grad-cam: Visual explanations from deep networks via gradient-based localization. In Proceedings of the IEEE international conference on computer vision. 618-626.

[25] Avanti Shrikumar, Peyton Greenside, and Anshul Kundaje. 2017. Learning important features through propagating activation differences. In Proceedings of the 34th International Conference on Machine Learning-Volume 70. JMLR. org, 3145-3153.

[26] Jost Tobias Springenberg, Alexey Dosovitskiy, Thomas Brox, and Martin Riedmiller. 2014. Striving for simplicity: The all convolutional net. arXiv preprint arXiv:1412.6806 (2014).

[27] Zhiguang Wang, Weizhong Yan, and Tim Oates. 2017. Time series classification from scratch with deep neural networks: A strong baseline. In 2017 International joint conference on neural networks (IFCNN). IEEE, 1578-1585.

[28] Sijie Yan, Yuanjun Xiong, and Dahua Lin. 2018. Spatial temporal graph convolutional networks for skeleton-based action recognition. In Thirty-second AAAI conference on artificial intelligence. 